\title{
Recent highlights with baryons from lattice QCD
}

\author{
Colin Morningstar ${ }^{1, *}$ \\ ${ }^{1}$ Department of Physics, Carnegie Mellon University, Pittsburgh, PA 15213, USA
}

\begin{abstract}
Highlights from recent computations in lattice QCD involving baryons are presented. Calculations of the proton mass and spin decompositions are discussed, a percent level determination of the nucleon axial coupling is described, and determinations of the proton and neutron electromagnetic form factors and light-cone parton distribution functions are outlined. Recent results applying the so-called Lüscher method to meson-baryon systems are presented. Key points emphasized are that much better precision with disconnected diagrams is being achieved, incorporating multi-hadron operators is now feasible, and more and more studies are being done with physical quark masses.
\end{abstract}

\section{Introduction}

Highlights from recent computations in lattice QCD involving baryons are presented in this talk. First, some introductory information about how baryons can be studied in lattice QCD is presented. Calculations of the proton mass and spin decompositions are then discussed, a percent level determination of the nucleon axial coupling is described, and determinations of the proton and neutron electromagnetic form factors and light-cone parton distribution functions are outlined. A current approach, involving the so-called Lüscher method, to confronting the challenge of studying baryon scattering and resonance properties in lattice QCD is then discussed. Some recent results applying the Lüscher method to meson-baryon systems are presented. A new calculation of the timelike pion form factor is highlighted to motivate future baryon form factor computations, especially the form factors for the $\Delta$ baryon which will be important to neutrino experiments such as DUNE. A new development with baryon-baryon interactions with the HAL QCD method is highlighted, and an $H$-dibaryon warm up calculation is presented. Key points emphasized are that much better precision with disconnected diagrams is being achieved, incorporating multihadron operators is now feasible, and more and more studies are being done with physical quark masses.

\section{Studying baryons in lattice QCD}

Finite-volume stationary-state energies are obtained in lattice QCD from Monte Carlo estimates of an $N \times N$ Hermitian correlation matrix

$$
C_{i j}(t)=\left\langle 0\left|O_{i}\left(t+t_{0}\right) \bar{O}_{j}\left(t_{0}\right)\right| 0\right\rangle .
$$

Crucial to the success in extracting the energies is the use of judiciously designed operators $\bar{O}_{j}$ to create the states of

*e-mail: cmorning@andrew.cmu.edu interest

$$
O_{j}(t)=O_{j}[\bar{\psi}(t), \psi(t), U(t)] .
$$

Temporal correlator estimates are obtained from path integrals over the quark $\psi, \bar{\psi}$ and gluon $U$ fields

$C_{i j}(t)=\frac{\int \mathcal{D}(\bar{\psi}, \psi, U) O_{i}\left(t+t_{0}\right) \bar{O}_{j}\left(t_{0}\right) \exp (-S[\bar{\psi}, \psi, U])}{\int \mathcal{D}(\bar{\psi}, \psi, U) \exp (-S[\bar{\psi}, \psi, U])}$

which involve the QCD action in imaginary time, formulated on a space-time lattice

$$
S_{\mathrm{QCD}}[\bar{\psi}, \psi, U]=\bar{\psi} K[U] \psi+S_{G}[U],
$$

where $K[U]$ is the quark Dirac matrix and $S_{G}[U]$ is the gluon action. The integrals over the Grassmann-valued quark fields are done exactly, bringing the correlators into forms such as

$$
C_{i j}(t)=\frac{\int \mathcal{D} U \operatorname{det} K[U] f\left(K^{-1}[U], \cdots, K^{-1}[U]\right) e^{-S_{G}[U]}}{\int \mathcal{D} U \operatorname{det} K[U] e^{-S_{G}[U]}}
$$

where $K^{-1}[U]$ are the quark propagators in the gluon field $U$. The remaining integrals over the gluon fields must be done with the Monte Carlo method, which uses a Markov chain to generate a sequence of gauge-field configurations $U_{1}, U_{2}, \ldots, U_{N}$. Including det $K$ in the Monte Carlo updating and evaluating the $K^{-1}$ in the numerator are the most computationally demanding parts of the calculation usually. The $\operatorname{det} K$ is handled by writing it as a multivariate Gaussian integral involving $K^{-1}$. A Metropolis method is employed with a sophisticated global updating proposal: Hybrid Monte Carlo (HMC) for the $u, d$ quarks, and Rational Hybrid Monte Carlo (RHMC) for the $s, c$ quarks.

Usually many different hadron operators are needed, so it is efficient to assemble them using basic building blocks, which we choose to be covariantly-displaced LapH-smeared[1] quark fields:

$$
q_{a \alpha j}^{A}=D^{(j)} \widetilde{\psi}_{a \alpha}^{(A)}, \quad \bar{q}_{a \alpha j}^{A}=\widetilde{\bar{\psi}}_{a \alpha}^{(A)} \gamma_{4} D^{(j) \dagger}
$$




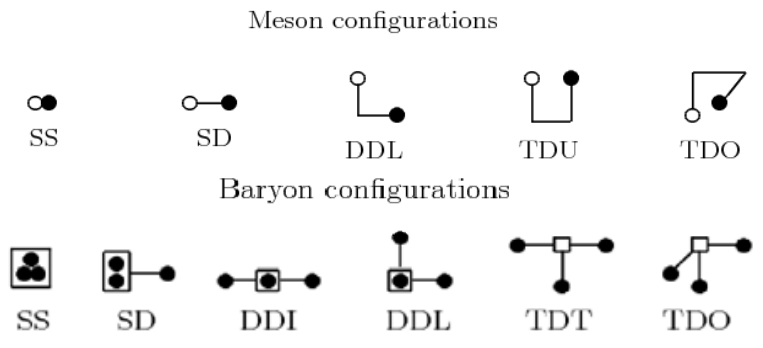

Figure 1. Spatial configurations of the meson and baryon operators in terms of displaced quark fields that we use. SS stands for single site, SD for singly displaced, DDL for doubly displaced in an L configuration, TDO for triply displaced orthogonal, and so on.

where the LapH-smeared quark field is

$$
\widetilde{\psi}_{a \alpha}(x)=\mathcal{S}_{a b}(x, y) \psi_{b \alpha}(y), \quad \mathcal{S}=\Theta\left(\sigma_{s}^{2}+\widetilde{\Delta}\right),
$$

and each displacement $D^{(j)}$ is a product of smeared links:

$$
D^{(j)}\left(x, x^{\prime}\right)=\widetilde{U}_{j_{1}}(x) \widetilde{U}_{j_{2}}\left(x+d_{1}\right) \ldots \widetilde{U}_{j_{p}}\left(x+d_{p-1}\right) \delta_{x^{\prime}, x+d_{p}} .
$$

Above, the $a, b$ are color indices, $\alpha$ is a Dirac spin index, $\sigma_{s}^{2}$ is the smearing cutoff, and $\widetilde{\Delta}$ is the three-dimensional gauge-covariant Laplacian. The gauge-covariant displacements utilize stout link[2] variables $\widetilde{U}_{k}(x)$. To a good approximation, the LapH smearing operator is $\mathcal{S}=V_{s} V_{s}^{\dagger}$ where the columns of matrix $V_{s}$ are the eigenvectors of $\Delta$ on each time slice.

The quark displacements build up the orbital and radial structures of the mesons and baryons. The spatial configurations we use are shown in Fig. 1. So-called elemental meson and baryon operators are then given by

$$
\begin{aligned}
\bar{\Phi}_{\alpha \beta}^{A B}(\boldsymbol{p}, t) & =\sum_{\boldsymbol{x}} e^{i \boldsymbol{p} \cdot\left(\mathbf{x}+\frac{1}{2}\left(\boldsymbol{d}_{\alpha}+\boldsymbol{d}_{\beta}\right)\right)} \delta_{a b} \bar{q}_{b \beta}^{B}(\boldsymbol{x}, t) q_{a \alpha}^{A}(\boldsymbol{x}, t), \\
\bar{\Phi}_{\alpha \beta \gamma}^{A B C}(\boldsymbol{p}, t) & =\sum_{\boldsymbol{x}} e^{i \boldsymbol{p} \cdot \mathbf{x}} \varepsilon_{a b c} \bar{q}_{c \gamma}^{C}(\boldsymbol{x}, t) \bar{q}_{b \beta}^{B}(\boldsymbol{x}, t) \bar{q}_{a \alpha}^{A}(\boldsymbol{x}, t) .
\end{aligned}
$$

Group-theory projections onto the irreps of the lattice symmetry group

$$
\bar{M}_{l}(t)=c_{\alpha \beta}^{(l) *} \bar{\Phi}_{\alpha \beta}^{A B}(t), \quad \bar{B}_{l}(t)=c_{\alpha \beta \gamma}^{(l) *} \bar{\Phi}_{\alpha \beta \gamma}^{A B C}(t),
$$

then produce meson $\bar{M}_{l}(t)$ and baryon $\bar{B}_{l}(t)$ operators which create states of definite momentum $\boldsymbol{p}$ in the irreps of the little group of $\boldsymbol{p}$.

The low-lying QCD mass spectrum has been successfully determined (see, for example, Ref. [3]), with a level of precision such that isospin breaking is now relevant. The next challenge now is to evaluate scattering amplitudes and extract resonance information.

For matrix element calculations, the standard method requires evaluating 3-point functions, such as those shown in Fig. 2. A major issue is ensuring the removal of excitedstate contamination by taking $t_{\text {sep }}, t_{\text {ins }}$, and $t_{\text {sep }}-t_{\text {ins }}$ large. In practice, this is difficult to achieve due to the signal-tonoise ratio which decreases with time separation. Computing so-called disconnected contributions, as shown on
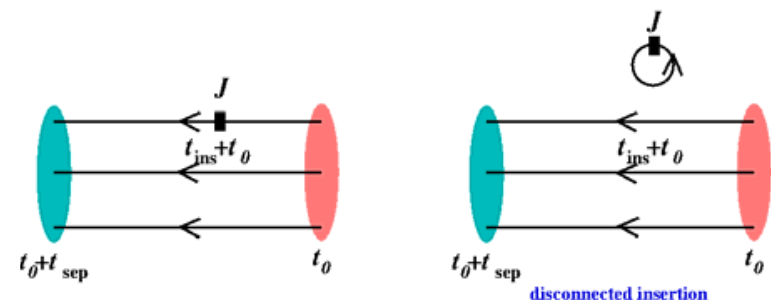

Figure 2. Diagrammatic representations of contributions to 3 point functions. (Left) The external current $J$ occurs on one of the three quark lines connecting the baryon source (pink) to the baryon sink (blue). (Right) So-called disconnected contributions in which the insertion of the current $J$ does not connect to the three quark lines between the baryon source and sink, but is involved in a separate sea quark loop.

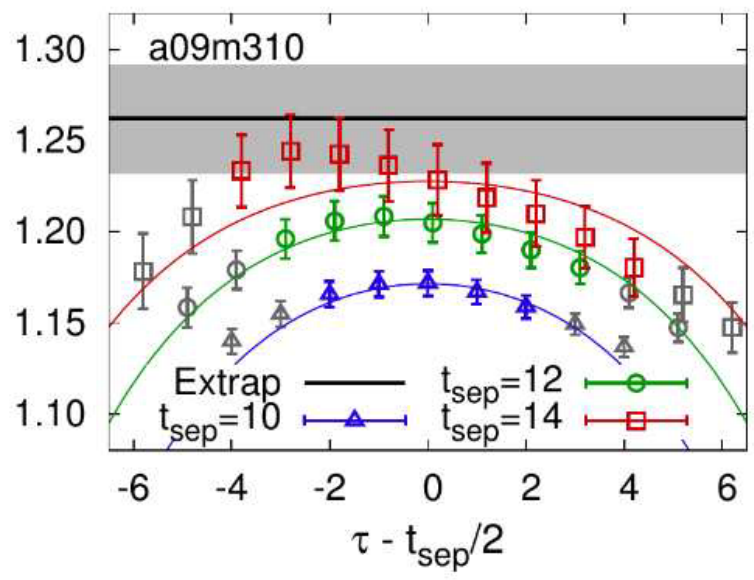

Figure 3. $g_{A}^{u-d}$ extractions for a variety of $t_{\mathrm{sep}}$, the time separation between the source and sink nucleon, and $\tau=t_{\text {ins }}$, the time where the current operator is inserted. Removal of excited-state contamination requires extrapolating to large $t_{\text {sep }}$ and looking for insensitivity in $\tau$ around $t_{\mathrm{sep}} / 2$. Results are from Ref. [8].

the right in Fig. 2, is much more difficult than the connected contributions, especially as the quark mass is taken to its physical value. Many previous studies simply neglected such contributions. However, a variety of new techniques are available now and such disconnected contributions are being reliably estimated with unprecedented precision. Our group uses the stochastic LapH method, whereas the results shown in Secs. 3-7 obtained by other groups use different methods, as mentioned in each case. Another issue with matrix element calculations is that the current operators require renormalization for comparison to $\overline{\mathrm{MS}}$. Such renormalizations can be evaluated using nonperturbative and/or perturbative methods.

Difficulties in removing excited-state contamination in calculations related to $g_{A}$ are illustrated in Fig. 3. The gray band shows the results of an extrapolation of the $t_{\text {sep }}=10,12,14$ results. It is also necessary to ensure insensitivity of the extrapolated results to the insertion time, which must be far from the source and sink. 


\section{Proton mass decomposition}

A recent determination of the mass decomposition of the proton has been presented in Ref. [4]. The rest mass $M$ of the proton is given by[5]

$$
M=-\left\langle T_{44}\right\rangle=\left\langle H_{m}\right\rangle+\left\langle H_{E}\right\rangle(\mu)+\left\langle H_{g}\right\rangle(\mu)+\frac{1}{4}\left\langle H_{a}\right\rangle,
$$

where $\left\langle T_{\mu \nu}\right\rangle$ is the expectation value of the energy momentum tensor in a hadron, and

$$
\begin{aligned}
& H_{m}=\sum_{u, d, s \cdots} \int d^{3} x m \bar{\psi} \psi, \\
& H_{E}=\sum_{u, d, s . \ldots} \int d^{3} x \bar{\psi}(\vec{D} \cdot \vec{\gamma}) \psi, \\
& H_{g}=\int d^{3} x \frac{1}{2}\left(B^{2}-E^{2}\right), \\
& H_{a}=\sum_{u, d, s \cdots} \int d^{3} x \gamma_{m} m \bar{\psi} \psi-\int d^{3} x \frac{\beta(g)}{g}\left(E^{2}+B^{2}\right),
\end{aligned}
$$

where $H_{m}$ is the quark condensate, $H_{E}$ is the quark energy, $H_{g}$ is the gluon field energy, and $H_{a}$ is the anomaly term. Note that $\left\langle H_{m}\right\rangle,\left\langle H_{a}\right\rangle,\left\langle H_{E}+H_{g}\right\rangle$ are scale and scheme independent. This study obtained the quark and gluon energies from the renormalized quark and gluon momentum fractions

$$
\left\langle H_{g}\right\rangle=\frac{3}{4} M\langle x\rangle_{g}, \quad\left\langle H_{E}\right\rangle=\frac{3}{4} M\langle x\rangle_{q}-\frac{3}{4}\left\langle H_{m}\right\rangle,
$$

and the anomaly term from $\left\langle H_{a}\right\rangle=M-\left\langle H_{m}\right\rangle$. The mass $M$ was determined from the two-point correlator, as usual, and a previous determination of $\left\langle H_{m}\right\rangle$ by these authors was used. The momentum fractions were evaluated using

$$
\begin{aligned}
\langle x\rangle_{q, g} & \equiv-\frac{\left\langle N\left|\frac{4}{3} \bar{T}_{44}^{q, g}\right| N\right\rangle}{M\langle N \mid N\rangle}, \\
\bar{T}_{44}^{q} & =\int d^{3} x \bar{\psi}(x) \frac{1}{2}\left(\gamma_{4} \overleftrightarrow{D}_{4}-\frac{1}{4} \sum_{i=0,1,2,3} \gamma_{i} \overleftrightarrow{D}_{i}\right) \psi(x), \\
\bar{T}_{44}^{g} & =\int d^{3} x \frac{1}{2}\left(E(x)^{2}-B(x)^{2}\right),
\end{aligned}
$$

taking renormalization into account:

$$
\begin{aligned}
\langle x\rangle_{u, d, s}^{R} & =Z_{Q Q}^{\overline{\mathrm{MS}}}(\mu)\langle x\rangle_{u, d, s}+\delta Z_{Q Q}^{\overline{\mathrm{MS}}}(\mu) \sum_{q=u, d, s}\langle x\rangle_{q}+Z_{Q G}^{\overline{\mathrm{MS}}}(\mu)\langle x\rangle_{g}, \\
\langle x\rangle_{g}^{R} & =Z_{G Q}^{\overline{\mathrm{MS}}}(\mu) \sum_{q=u, d, s}\langle x\rangle_{q}+Z_{G G}^{\overline{\mathrm{MS}}}\langle x\rangle_{g} .
\end{aligned}
$$

Results were obtained on four ensembles using an $N_{f}=$ $2+1$ domain-wall fermion action with overlap valence propagators. The difficult disconnected insertions employed cluster-decomposition error reduction with all time slices looped over. Extrapolations to remove systematic errors were done with a global fit including finite-volume and finite-spacing corrections, as well as known chiral behavior. The impressive results are shown in Fig. 4 and can be summarized as quark energy 32(4)(4)\%, glue energy $36(5)(4) \%$, quark condensate $9(2)(1) \%$, and trace anomaly 23(1)(1)\%.

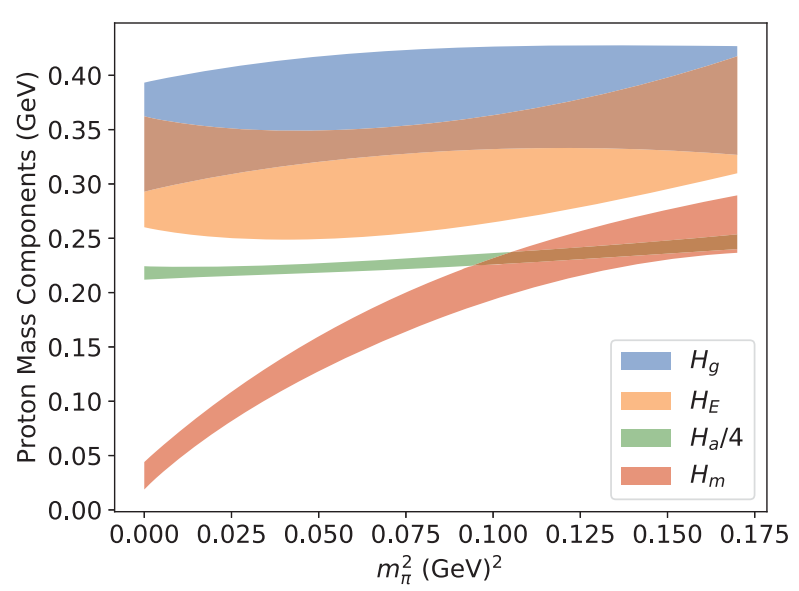

Figure 4. Proton mass decomposition from Ref. [4] against the pion mass squared. $H_{g}$ is the gluon field energy, $H_{E}$ is the quark energy, $H_{a}$ is the anomaly term, and $H_{m}$ is the quark condensate.

\section{Nucleon spin decomposition}

A recent determination of the spin and momentum fraction decomposition of the nucleon has been presented in Ref. [6]. From the Ji sum rule[7], the nucleon spin is given by

$$
J_{N}=\sum_{q=u, d, s, c \cdots}\left(\frac{1}{2} \Delta \Sigma_{q}+L_{q}\right)+J_{g}=\sum_{q} J_{q}+J_{g}
$$

where $J_{g}$ is the gluon total angular momentum, $L_{q}$ is the quark orbital angular momentum, and $\frac{1}{2} \Delta \Sigma_{q}$ is the contribution from the intrinsic quark spin. These quantities were obtained from the following nucleon matrix elements, with $Q=p^{\prime}-p$ and $P=\frac{1}{2}\left(p^{\prime}+p\right)$ :

$$
\begin{aligned}
\left\langle N\left(p, s^{\prime}\right)\left|\bar{q} \gamma_{\mu} \gamma_{5} q\right| N(p, s)\right\rangle & =\bar{u}_{N}\left(p, s^{\prime}\right)\left[g_{A}^{q} \gamma^{\mu} \gamma_{5}\right] u_{N}(p, s), \\
\left\langle N\left(p^{\prime}, s^{\prime}\right)\left|\bar{q} \gamma^{\{\mu} \overleftrightarrow{D^{v}}{ }^{\gamma} q\right| N(p, s)\right\rangle & =\bar{u}_{N}\left(p^{\prime}, s^{\prime}\right) \Lambda_{\mu \nu}^{q}\left(Q^{2}\right) u_{N}(p, s), \\
\Lambda_{q(g)}^{\mu \nu}\left(Q^{2}\right)=A_{20}^{q(g)}\left(Q^{2}\right) \gamma^{\{\mu} P^{\nu\}} & +B_{20}^{q(g)}\left(Q^{2}\right) \frac{\sigma^{\{\mu \alpha} q_{\alpha} P^{\nu\}}}{2 m} \\
& +C_{20}^{q(g)}\left(Q^{2}\right) \frac{1}{m} Q^{\{\mu} Q^{\nu\}} .
\end{aligned}
$$

The quark(gluon) total angular momentum and quark momentum fraction and spin were extracted using

$$
\begin{aligned}
J_{q(g)} & =\frac{1}{2}\left[A_{20}^{q(g)}(0)+B_{20}^{q(g)}(0)\right], \\
\langle x\rangle_{q} & =A_{20}^{q}(0), \quad \Delta \Sigma_{q}=g_{A}^{q},
\end{aligned}
$$

and the gluon momentum fraction was obtained from $O_{\mu \nu}^{g}=2 \operatorname{Tr}\left[G_{\mu \sigma} G_{\nu \sigma}\right]$ with $\bar{O}^{g} \equiv O_{44}^{g}-\frac{1}{3} O_{j j}^{g}$, and

$$
\left\langle N\left(p, s^{\prime}\right)\left|\bar{O}^{g}\right| N(p, s)\right\rangle=\left(-4 E_{N}^{2}-\frac{2}{3} \vec{p}^{2}\right)\langle x\rangle_{g} .
$$

One ensemble at the physical point on a $48^{3} \times 96$ lattice using a twisted mass clover-improved action with lattice spacing $a=0.0939(3) \mathrm{fm}$, set from the nucleon mass, was used. The $u, d$ disconnected diagrams were estimated 

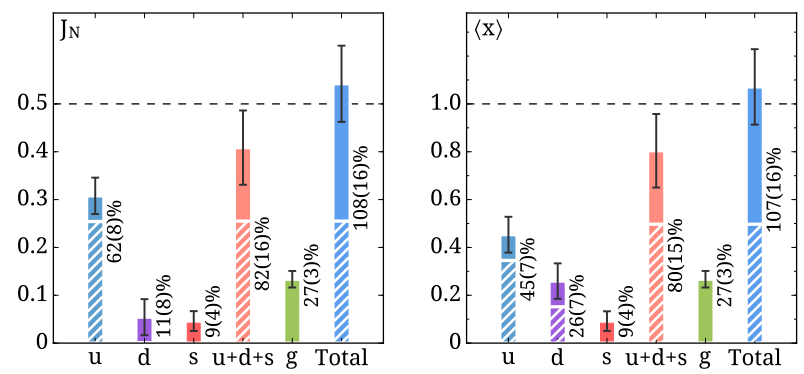

Figure 5. Nucleon spin (left) and momentum fraction (right) decompositions from Ref. [6] in terms of the contributions from each quark flavor and from gluons. Striped segments refer to valence quark contributions, while solid segments show sea quark and gluon contributions.

by exact deflation plus the one-end-trick, while the $s$ disconnected diagrams were evaluated by a truncated solver method. Renormalization factors were determined nonperturbatively. Their final results are shown in Fig. 5.

\section{Nucleon axial coupling}

A remarkable percent level determination of the nucleon axial coupling $g_{A}$ in lattice QCD recently appeared[8]. The use of a Feynman-Hellman method enabled a significant reduction in statistical errors. Their value was

$$
g_{A}=1.2711(103)^{s}(39)^{\chi}(15)^{a}(19)^{V}(04)^{I}(55)^{M},
$$

where the errors, in order, are from the statistical estimation, chiral extrapolation, lattice spacing extrapolation, volume extrapolations, isospin corrections, and model selection. Extrapolations were done using several models and the final estimate is a model average. Sixteen ensembles from the MILC collaboration generated using a HISQ action with lattice spacings $a \sim 0.15 \mathrm{fm}, a \sim 0.12 \mathrm{fm}$, and $a \sim 0.09 \mathrm{fm}$ and pion masses ranging from near physical to $400 \mathrm{MeV}$ were used. Domain-wall valence propagators were used, making this a mixed-action computation. Their main result is illustrated in Fig. 6, and a comparison of their estimate with other recent determinations is shown in Fig. 7.

\section{Proton/neutron electromagnetic form factors}

A recent study of the proton and neutron electromagnetic form factors in lattice QCD was presented in Ref. [9]. One ensemble using an $N_{f}=2+1+1$ twisted mass action with $m_{\pi}=130 \mathrm{MeV}$, and two ensembles using an $N_{f}=2$ twisted mass action with $m_{\pi}=130 \mathrm{MeV}$ and two volumes $L m_{\pi} \sim 3$ and $L m_{\pi} \sim 4$ were utilized. An unprecedented precision of the disconnected diagram contributions was achieved using hierarchical probing, low mode deflation, and large numbers of smeared point sources to reduce gauge noise. The results demonstrated that the disconnected diagrams have nonnegligible effects. The study

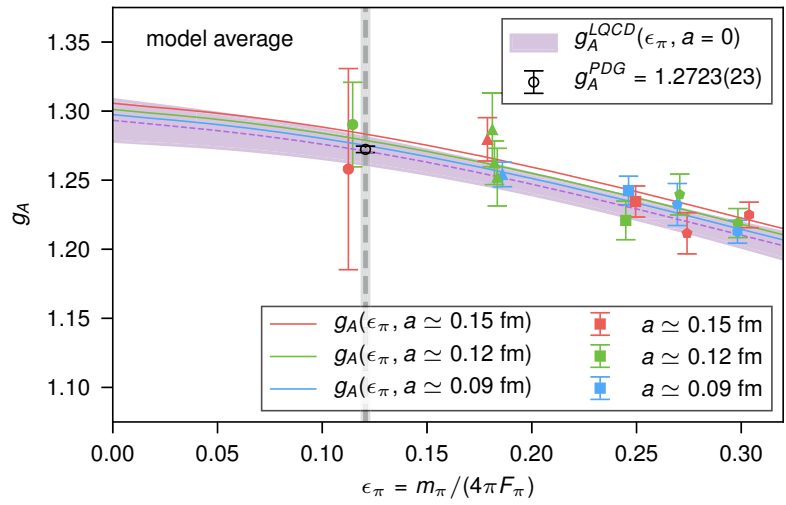

Figure 6. Extrapolation of a $g_{A}$ determination in Ref. [8] to the physical point. Solid red, green, and blue curves are central values of $g_{A}$ as a function of $\epsilon_{\pi}=m_{\pi} /\left(4 \pi F_{\pi}\right)$ at fixed lattice spacing and infinite volume, and the black circle is the experimental value. Magenta band is the central $68 \%$ confidence band of the continuum and infinite volume extrapolations.

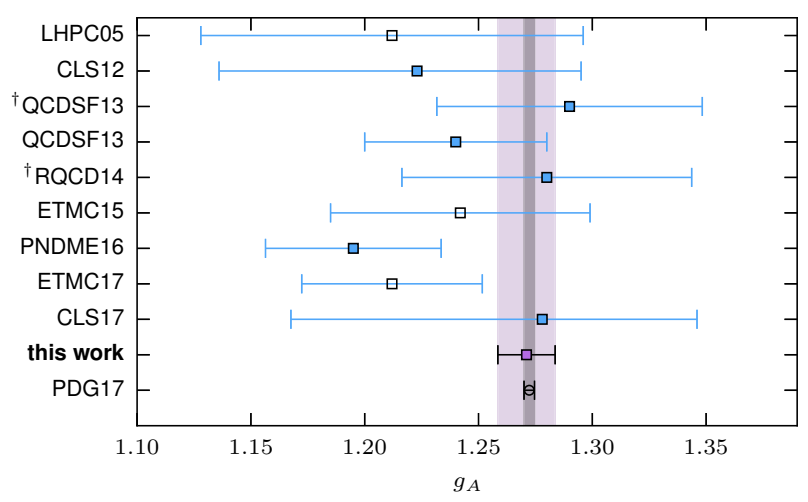

Figure 7. Comparison of the estimate of $g_{A}$ from Ref. [8] with other recent determinations. See Ref. [8] for references to the other estimates. Results with closed symbols have included an extrapolation to the continuum limit, while results with open symbols have only included extrapolation to the physical pion mass. To guide the eye, the vertical magenta band is the full uncertainty from Ref. [8], while the vertical gray band is the experimental uncertainty.

included a thorough investigation of excited-state contamination, but further study of finite-volume effects at low $Q^{2}$ is needed. Their $N_{f}=2+1+1$ results are compared to experiment in Fig. 8.

\section{Light-cone parton distribution function}

A first determination of the unpolarized helicity parton distribution function (PDF) at the physical point with nonperturbative renormalization and large momenta treated was presented in Ref. [10]. Extracting PDFs from their moments is impractical, so these authors used a clever method proposed by Ji[11] with subsequent refinements. First, they computed spatial correlations between boosted nucleon states, then carried out Fourier transforms to produce quantities known as quasi-PDFs, then finally took the 

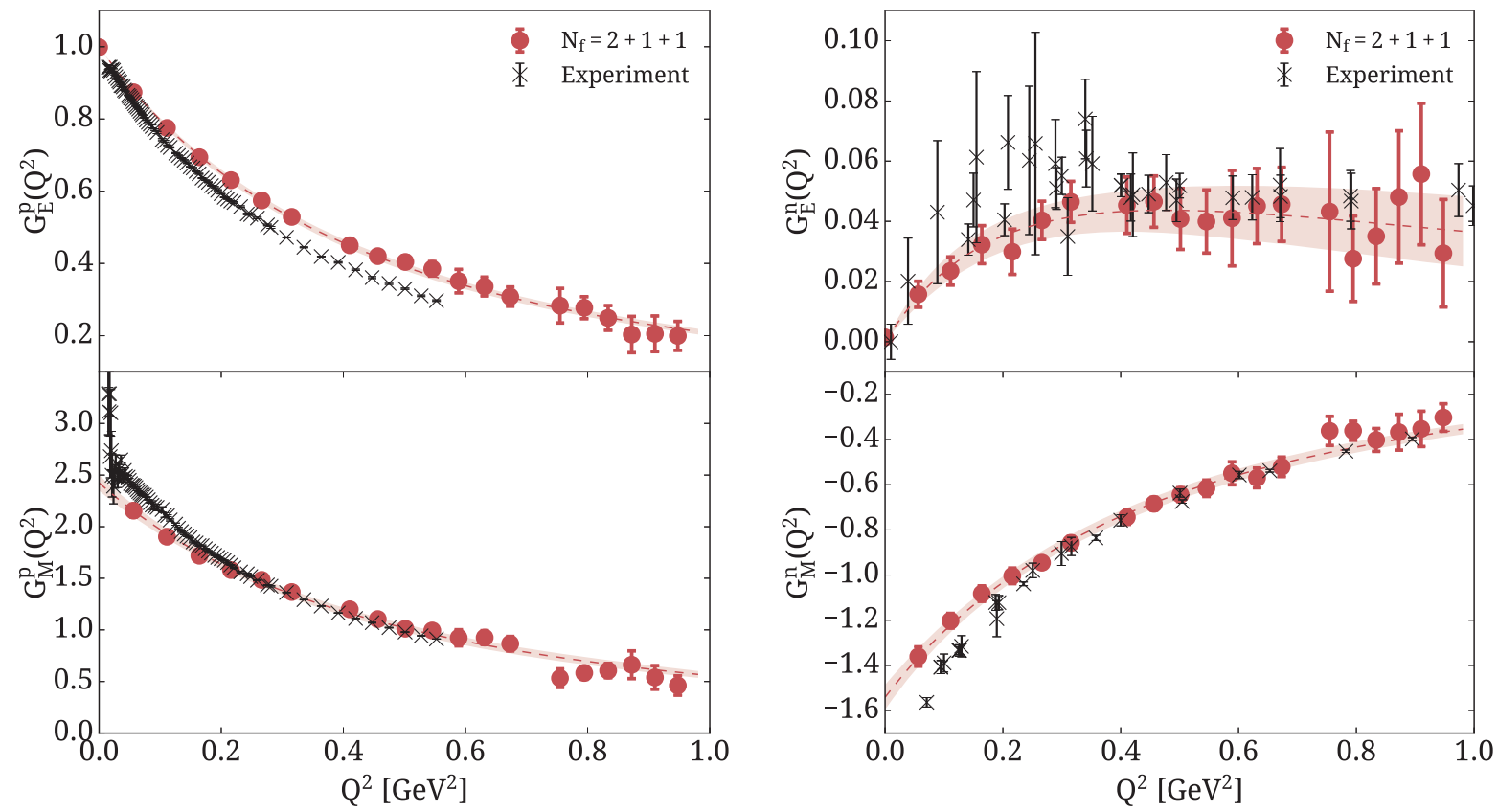

Figure 8. Electric (top) and magnetic (bottom) form factors of the proton (left) and neutron (right) from Ref. [9], compared to experiment.

infinite-momentum limit via a refined matching procedure. So-called target mass corrections were employed, as well as a renormalization scheme for the Wilson line operators.

Results were obtained on one ensemble using a $48^{3} \times$ 96 lattice with a twisted mass $N_{f}=2$ action with lattice spacing $a=0.0938(3)(2)$ fm and $m_{\pi} L=2.98(1)$ at the physical point. Both unpolarized and polarized PDFs for three momenta were compared to some phenomenological curves, shown in Fig. 9. Improvement of these estimates of the PDFs is ongoing.

\section{Excited baryon states}

In finite volume, the stationary-state energies are discrete due to momentum quantization, so temporal correlation matrices have the spectral representation

$$
C_{i j}(t)=\sum_{n} Z_{i}^{(n)} Z_{j}^{(n) *} e^{-E_{n} t}, \quad Z_{j}^{(n)}=\left\langle 0\left|O_{j}\right| n\right\rangle,
$$

neglecting wrap-around corrections. It is not practical to do fits using the above form, so diagonalization methods with single- and two-exponential fits are employed to extract some number of low-lying states.

To access excited baryon (and meson) states reliably, it is necessary to extract the energies of the multi-hadron states that are lower-lying than the excited baryons of interest. This requires evaluating correlators involving multi-hadron operators. Good multi-hadron operators involve combining good individual hadron operators which separately have well-defined momenta. With such operators, the usual point-to-all trick, which exploits translational invariance, cannot be utilized to drastically reduce the number of quark propagator sources that are needed, so an overly large number of Dirac matrix inversions are required. One solution to this is the stochastic $\mathrm{LapH}$ method[1] which estimates the entire matrix inverse, or a large portion of it, using an additional Monte Carlo calculation, exploiting the Laplacian-Heaviside quark smearing and various noise dilution projectors for variance reduction.

Excited $\Lambda$ baryons were studied somewhat recently in Ref. [12]. A small sample of the results obtained is shown in Fig. 10.

\section{Scattering amplitudes from lattice QCD}

Finite-volume energies $E$ in lattice QCD are related to the infinite-volume $S$ matrix[13]. Utilizing such relations to obtain scattering amplitudes is often termed the Lüscher method. Introduce the $K$-matrix as usual,

$$
S=(1+i K)(1-i K)^{-1}=(1-i K)^{-1}(1+i K) .
$$

In the $J L S a$ basis, for total angular momentum $J$, orbital angular momentum $L$, intrinsic spin $S$, and species channel $a$, introduce

$$
K_{L^{\prime} S^{\prime} a^{\prime} ; L S a}^{-1}(E)=q_{\mathrm{cm}, a^{\prime}}^{-L^{\prime}-\frac{1}{2}} \widetilde{K}_{L^{\prime} S^{\prime} a^{\prime} ; L S a}^{-1}\left(E_{\mathrm{cm}}\right) q_{\mathrm{cm}, a}^{-L-\frac{1}{2}},
$$

then below 3-particle thresholds, there is a quantization condition

$$
\operatorname{det}\left(1-B^{(\boldsymbol{P})} \widetilde{K}\right)=\operatorname{det}\left(1-\widetilde{K} B^{(\boldsymbol{P})}\right)=0
$$

or

$$
\operatorname{det}\left(\widetilde{K}^{-1}-B^{(\boldsymbol{P})}\right)=0
$$

where the Hermitian "box matrix" $B^{(\boldsymbol{P})}$ encodes the effects of the cubic finite-volume. Details about the box matrix may be found in Ref. [14]. 

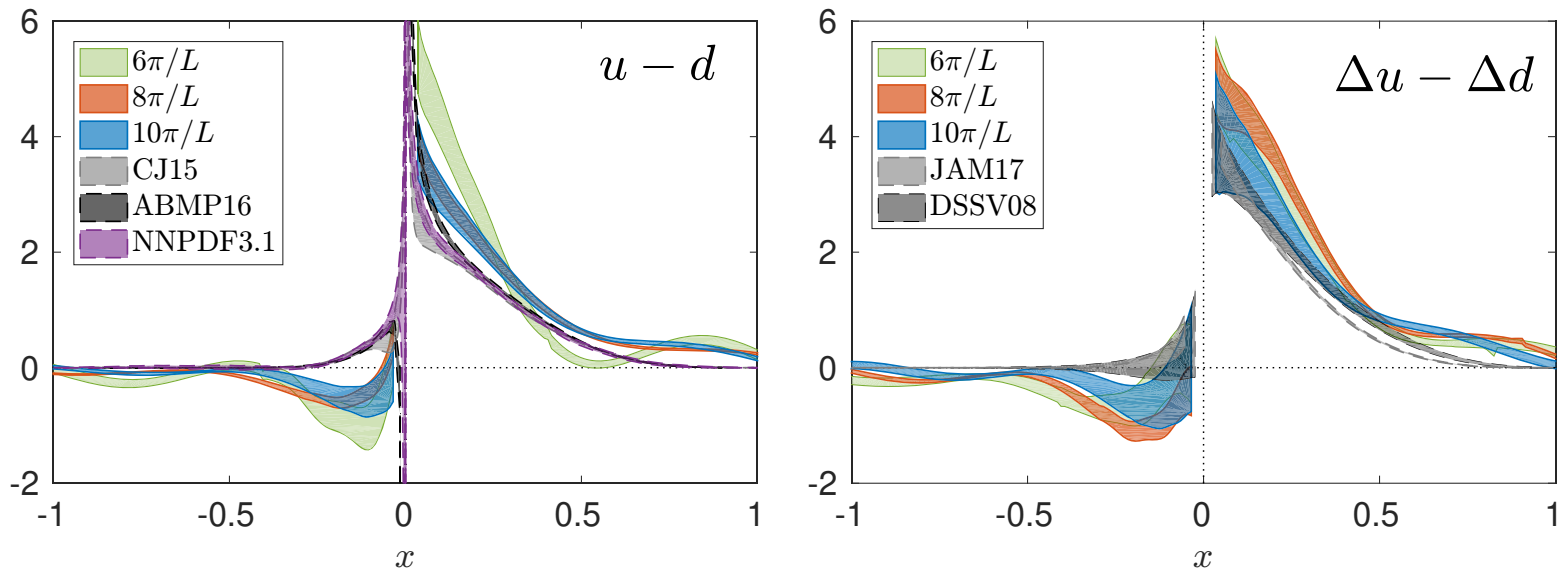

Figure 9. Unpolarized (left) and polarized (right) parton distribution functions for three momenta compared to some phenomenological curves. Results are from Ref. [10].
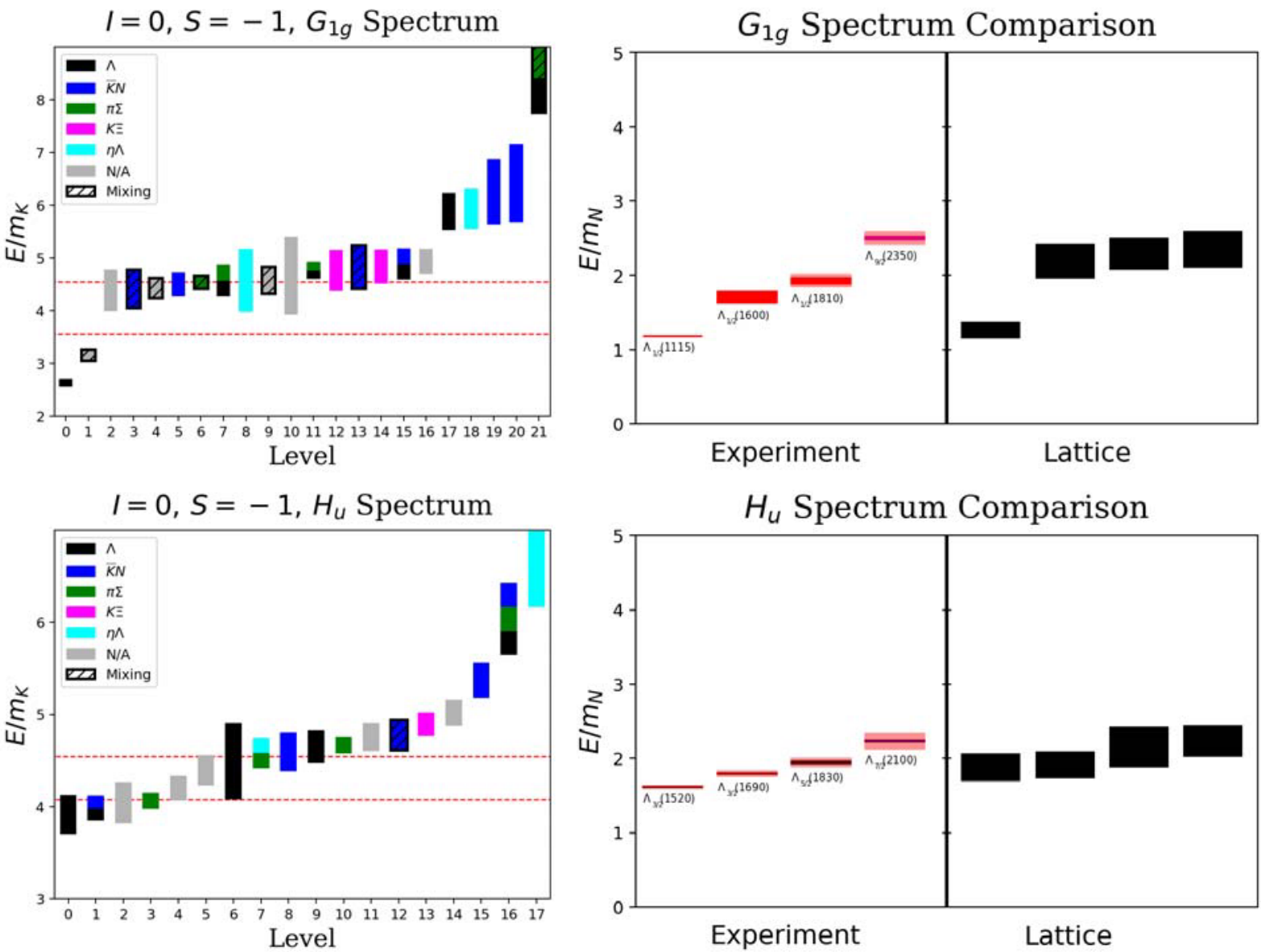

Figure 10. (Left) Stationary state energies in the $I=0, S=-1$ baryonic flavor sector for a $32^{3} \times 256$ anisotropic lattice with $m_{\pi} \sim 240 \mathrm{MeV}$. The $G_{1 g}$ channel (even parity containing the spin- $\frac{1}{2}$ states) and the $H_{u}$ channel (odd parity including the spin- $\frac{3}{2}$ states) are shown as ratios over the kaon mass $m_{K}$. Different colors indicate the different properties of the states as deduced by the fitted overlap factors $Z_{j}^{(n)}$. (Right) The single-baryon dominated levels are compared to experiment as ratios over the nucleon mass $m_{N}$. Results are from Ref. [12]. 


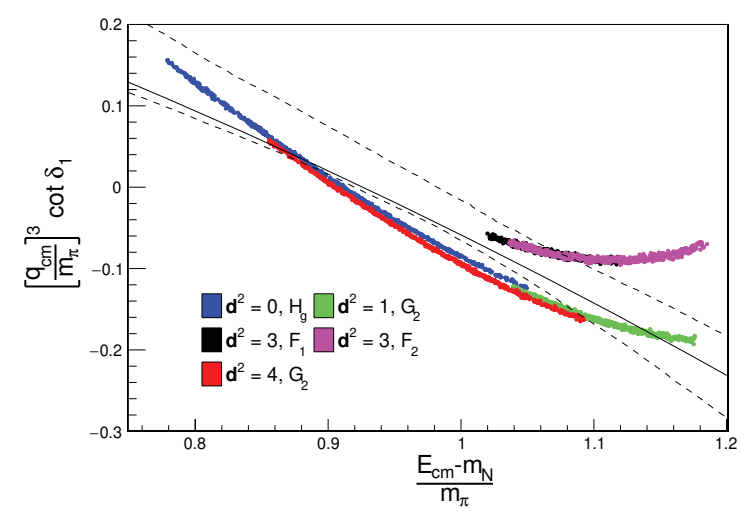

Figure 11. $P$-wave $N \pi$ scattering phase shift against center-ofmass energy showing the $\Delta$ resonance where the $\cot \delta_{1}$ crosses zero. Results are from Ref. [15].

The quantization condition relates a single energy $E$ to the entire $K$-matrix, so one cannot solve for the $K$ matrix, except in a single channel for a single partial wave. Thus, we must approximate the $K$-matrix with functions depending on a handful of fit parameters. We then obtain estimates of the fit parameters using many different energies. The quantization condition involves an infinitedimensional determinant. We make the condition practical by first transforming to a block-diagonal basis, then truncating in orbital angular momentum. Meson-meson scattering studies are becoming mature, whereas only a few meson-baryon scattering investigations have been attempted. Baryon-baryon scattering studies are currently gestating.

The decay $\Delta(1232) \rightarrow N \pi$ was recently studied in Ref. [15]. Only the $L=1$ partial wave was included. Results were obtained on a large $48^{3} \times 128$ isotropic lattice with $m_{\pi} \approx 280 \mathrm{MeV}$ and $a \sim 0.076 \mathrm{fm}$. Their determination of the scattering phase shift is shown in Fig. 11. A Breit-Wigner fit gave $m_{\Delta} / m_{\pi}=4.738(47)$ and $g_{\Delta N \pi}=$ 19.0(4.7). Note that experiment yields $g_{\Delta N \pi} \sim 16.9$.

Preliminary results for the $\Delta$ resonance in another recent study using a lattice with length $L=2.8 \mathrm{fm}$, spacing $a=0.116 \mathrm{fm}$ and pion mass $m_{\pi}=260 \mathrm{MeV}$ has appeared in Ref. [16]. No slice-to-slice propagators were used, three total momenta were studied, the ground and excited states were extracted, and their analysis included only a single partial wave. Their determination of the scattering phase shift is shown in Fig. 12.

Preliminary results from our most recent study[17] of the $\Delta$ resonance are shown in Fig. 13. The lattice length, lattice spacing, and pion mass are $L=4.2 \mathrm{fm}$, $a=0.065 \mathrm{fm}, m_{\pi}=200 \mathrm{MeV}$. Five total momenta have been used, and both ground and excited states were extracted. We expect to reduce statistical errors by a factor of 6 in our final results. The finite volume spectrum which produced these results is shown in Fig. 14. Fits included irreps which mix the $S$ and $P$ waves and relied on the automated determination of $B$-matrix elements from Ref. [14].

Results for $\Lambda(1405) \rightarrow \Sigma \pi$ will be presented in the near future in Ref. [18]. Very preliminary results on a lat-

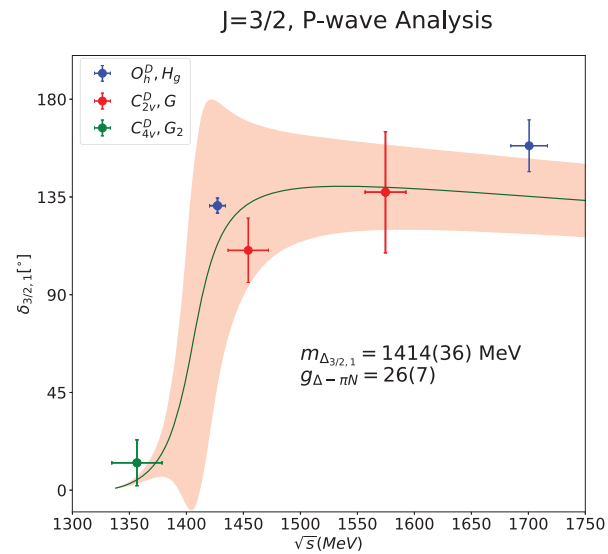

Figure 12. $P$-wave $N \pi$ scattering phase shift against the Mandelstam variable $\sqrt{s}$ showing the $\Delta$ resonance where the phase shift rises dramatically. Results are from Ref. [16].
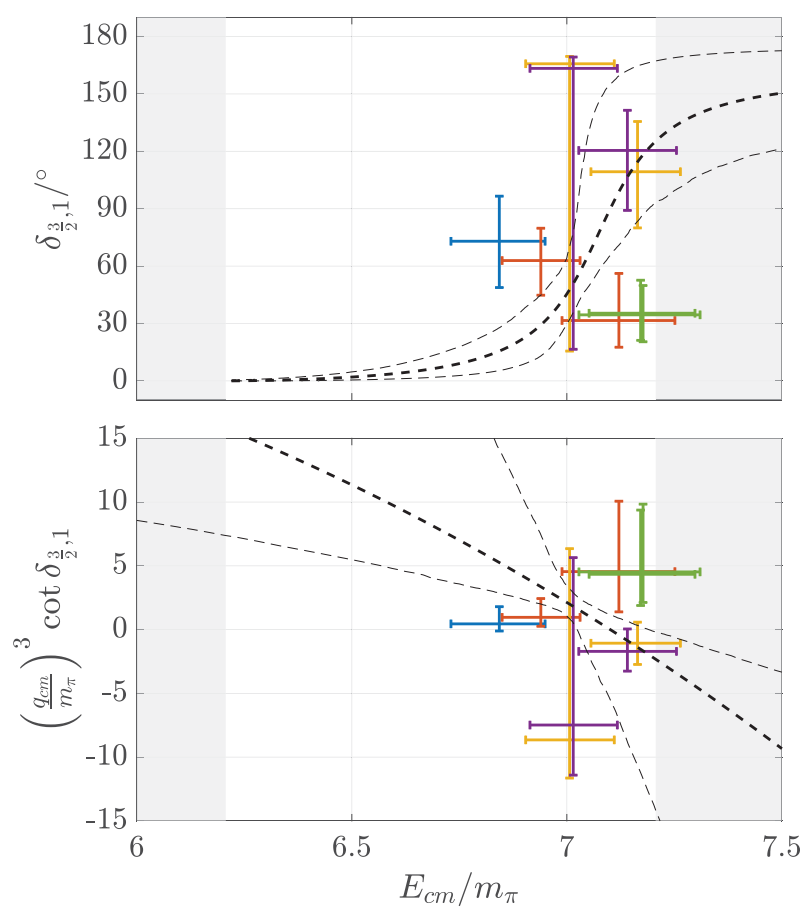

Figure 13. $N \pi$ scattering phase shifts near the $\Delta$ resonance from Ref. [17] against the center-of-mass energy as a ratio over the pion mass. The top plot shows the phase shift itself, and the bottom plot shows the cotangent of the phase shift multiplied by threshold factors.

tice with $L=3.2 \mathrm{fm}, a=0.065 \mathrm{fm}, m_{\pi}=280 \mathrm{MeV}$ are shown in Fig. 15.

\section{Three last items}

I finish this talk by reporting on three last items of interest. 


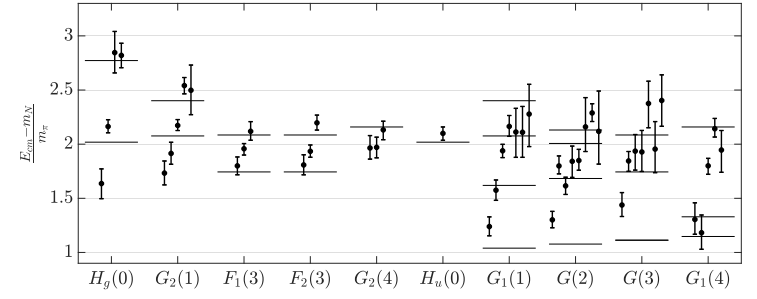

Figure 14. The energy spectrum of finite-volume states used in our most recent study in Ref. [17] of the $\Delta$ resonance. Differences of the center-of-mass energies from the nucleon mass as a ratio over the pion mass are shown for the different irreps and total momenta used. Horizontal lines show the non-interacting energies.

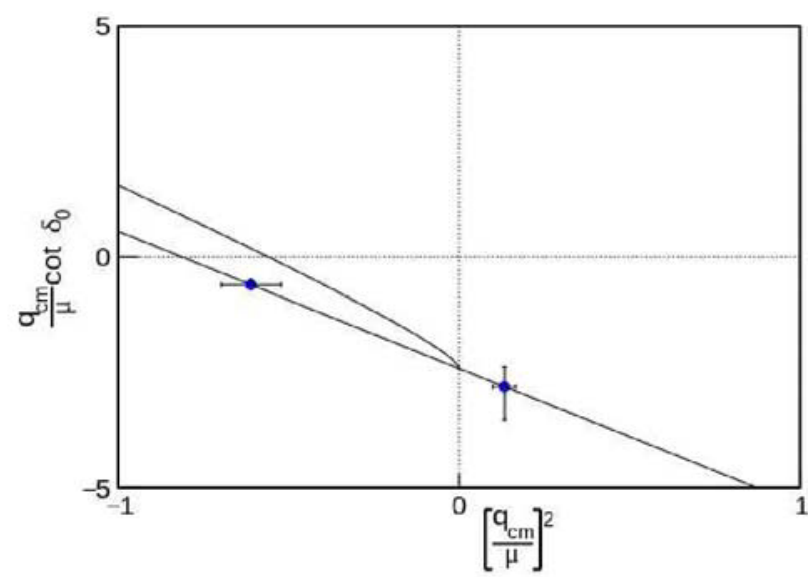

Figure 15. Very preliminary results for the $\Sigma \pi$ scattering phase shift near the $\Lambda(1405)$ resonance against the center-of-mass momentum as ratio with a reference energy $\mu$. Result are from Ref. [18].

A recent determination of the time-like pion form factor appeared in Ref. [19]. It was extracted using

$$
\begin{aligned}
\left|F_{\pi}\left(E_{\mathrm{cm}}\right)\right|^{2}= & g_{\Lambda}(\gamma)\left(q_{\mathrm{cm}} \frac{\partial \delta_{1}}{\partial q_{\mathrm{cm}}}+u \frac{\partial \phi_{1}^{(d, \Lambda)}}{\partial u}\right) \\
& \times \frac{3 \pi E_{\mathrm{cm}}^{2}}{2 q_{\mathrm{cm}}^{5} L^{3}}\left|\left\langle 0\left|V^{(\boldsymbol{d}, \Lambda)}\right| \boldsymbol{d} \Lambda n\right\rangle\right|^{2},
\end{aligned}
$$

where

$$
\gamma=\frac{E}{E_{\mathrm{cm}}}, \quad u=\frac{L q_{\mathrm{cm}}}{2 \pi}, \quad g_{\Lambda}(\gamma)= \begin{cases}\gamma^{-1}, & \Lambda=A_{1}^{+} \\ \gamma, & \text { otherwise }\end{cases}
$$

$\delta_{1}$ is the physical phase shift, and the pseudophase $\phi_{1}^{(d, \Lambda)}$ is obtained from $B_{11}^{(d, \Lambda)}=\left(q_{\mathrm{cm}} / m_{\pi}\right)^{3} \cot \phi_{1}^{(\boldsymbol{d}, \Lambda)}$. The matrix element

$$
V^{(\boldsymbol{d}, \Lambda)}=\sum_{\mu} b_{\mu}^{(\boldsymbol{d}, \Lambda)} V_{R, \mu}, \quad \sum_{\mu} b_{\mu}^{(\boldsymbol{d}, \Lambda) *} b_{\mu}^{(\boldsymbol{d}, \Lambda)}=1,
$$

with

$$
\begin{aligned}
V_{R, \mu} & =Z_{V}\left(1+a b_{V} m_{1}+a \bar{b}_{V} \operatorname{Tr} M_{q}\right) V_{I, \mu}, \\
V_{I, \mu} & =V_{\mu}+a c_{V} \widetilde{\partial}_{\nu} T_{\mu \nu}
\end{aligned}
$$

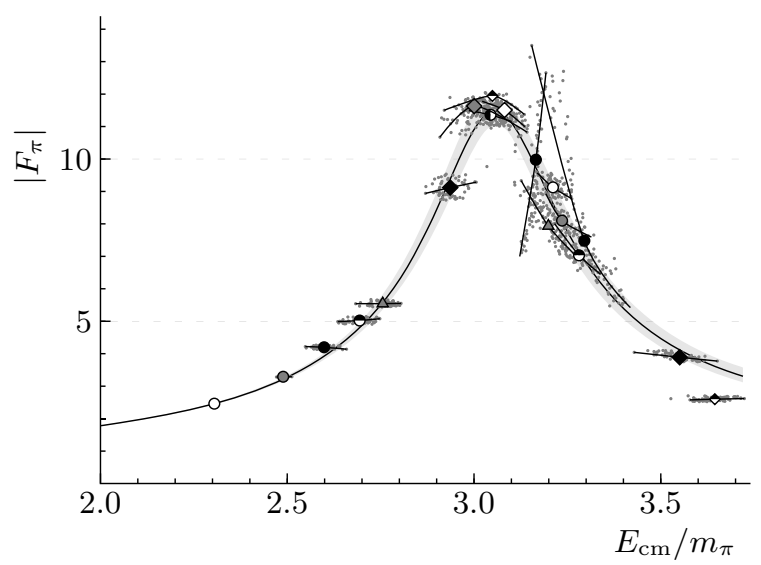

Figure 16. Timelike pion form factor $\left|F_{\pi}\right|$ again center-of-mass energy over pion mass $E_{\mathrm{cm}} / m_{\pi}$ using the CLS J303 ensemble on a $64^{3} \times 192$ lattice with spacing $a=0.050 \mathrm{fm}$ and $m_{\pi}=$ $260 \mathrm{MeV}$. The curve is a fit with a thrice-subtracted dispersion relation. Results are from Ref. [19]. This shows the feasibility of future calculations of baryon form factors.

and

$$
V_{\mu}^{a}=\frac{1}{2} \bar{\psi} \gamma_{\mu} \tau^{a} \psi, \quad \widetilde{\partial}_{\nu} T_{\mu \nu}^{a}=\frac{1}{2} i \widetilde{\partial}_{\nu} \bar{\psi} \sigma_{\mu \nu} \tau^{a} \psi
$$

was computed and used to determine the form factor. Results for CLS J303 ensemble on a $64^{3} \times 192$ lattice with spacing $a=0.050 \mathrm{fm}$ and $m_{\pi}=260 \mathrm{MeV}$ are shown in Fig. 16. The success of this calculation paves the way for baryon form factor determinations. A similar method is now being used for $\Delta$ transition form factors needed by the Deep Underground Neutrino Experiment (DUNE).

The HAL QCD collaboration has extensively studied nucleon-nucleon interactions. Their method extracts observables from non-local kernels associated with tempospatial correlation functions. However, a controversy arose when disagreements of their results with so-called direct methods were found. A recent study[20] suggests that this discrepany arises from the misidentification of energies in the direct method. The $\Xi \Xi\left({ }^{1} S_{0}\right)$ temporal correlation function was studied in detail, with pertinent results shown in Fig. 17. In the right plot in this figure, the key point is the disagreement between the dashed line (the known result) and the apparent plateau of the effective mass points in blue. The shaded blue band is the expected behavior of this function for larger time separations. Hopefully this resolution will accelerate progress in baryon-baryon scattering.

A recent report on an ongoing study of the $H$-dibaryon was presented in Ref. [21]. These results were obtained at the $S U(3)$ flavor symmetric point using well-designed baryon-baryon operators since a previous study showed that a hexaquark operator would not saturate the signal. They found several finite-volume energies below the $\Lambda \Lambda$ threshold. Some of their results are shown in Fig. 18. A scattering amplitude analysis is needed to determine if the system is bound or a resonance. Due to the small lattices used and the very heavy pion, this must be viewed 

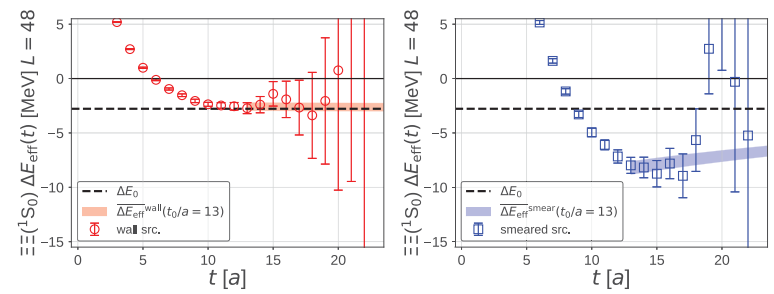

Figure 17. Effective masses associated with $\Xi \Xi\left({ }^{1} S_{0}\right)$ correlators using (left) a wall source and (right) a local smeared source. The dashed line indicates the asymptotic behavior using the wall source, and this result also agrees with the HAL QCD method. The key point, shown in the right plot, is the disagreement between the dashed line and the apparent plateau of the effective mass points in blue obtained using the local smeared source. The shaded blue band in the right plot is the expected large-time behavior. These results are from Ref. [20].

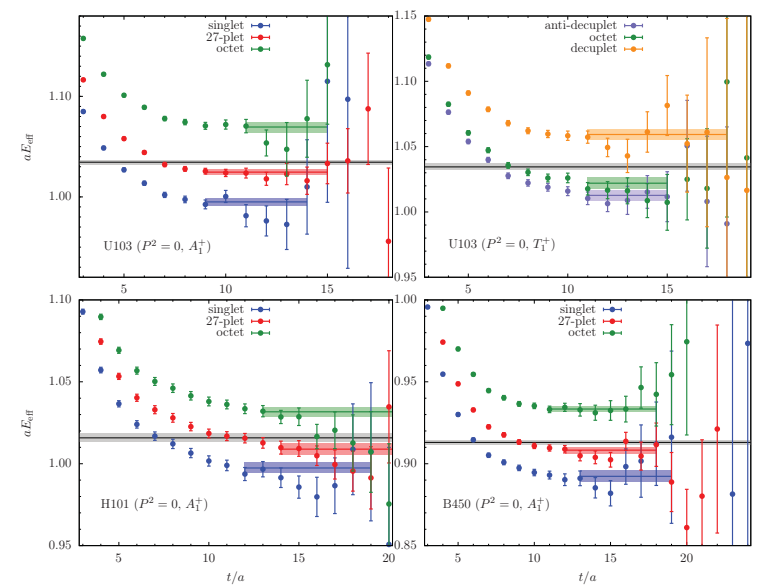

Figure 18. Effective masses for spin- 0 and spin- 1 dibaryon operators of different flavor irreps using 3 ensembles from Ref. [21]. Blue points show flavor singlet results, the red points show the flavor 27-plet, and the flavor octet is shown in green. Results on the CLS U103 ensemble in the $A_{1}^{+}$spin-zero irrep are shown in the upper left. The upper right plot shows results on the U103 ensemble for the $T_{1}^{+}$spin-one irrep. Results in the $A_{1}^{+}$spin-zero irrep on the H101 and B450 ensembles are shown in the lower left and lower right plots, respectively. Horizontal black lines show the two-octet baryon thresholds. The $S U(3)$ flavor symmetric point is used, and the lattice volumes are small.

as a warm up exercise. Future work on larger lattices and lighter pions will involve the stochastic LapH method.

\section{Conclusion}

Highlights from recent computations in lattice QCD involving baryons were presented in this talk. How baryons can be studied in lattice QCD was first discussed, followed by results on the proton mass and spin decompositions, nucleon axial coupling, the proton and neutron electromagnetic form factors, and light-cone parton distribution functions. Recent works on meson-baryon scattering using the so-called Lüscher method were shown. Key points emphasized were that much better precision with discon- nected diagrams is being achieved, incorporating multihadron operators is now feasible, and more and more studies are being done with physical quark masses.

\section{Acknowledgements}

Support from the U.S. National Science Foundation under award PHY-1613449 is gratefully acknowledged.

\section{References}

[1] C. Morningstar, J. Bulava, J. Foley, K.J. Juge, D. Lenkner, M. Peardon, C.H. Wong, Phys. Rev. D83, 114505 (2011).

[2] C. Morningstar and M. Peardon, Phys. Rev. D69, 054501 (2004).

[3] A. Kronfeld, Ann. Rev. Nucl.Part. Sci 62, 265 (2012).

[4] Y. Yang, J. Liang, Y. Bi, Y. Chen, T. Draper, K. F. Liu, Z. Liu, Phys. Rev. Lett. 121, 212001 (2018).

[5] X. Ji, Phys. Rev. Lett. 74, 1071 (1995).

[6] C. Alexandrou, M. Constantinou, K. Hadjiyiannakou, K. Jansen, C. Kallidonis, G. Koutsou, A. V. AvilésCasco, C. Wiese, Phys. Rev. Lett. 119, 142002 (2017).

[7] X. Ji, Phys. Rev. Lett. 78, 610 (1997).

[8] C.Chang et al., Nature 558, 91 (2018); arXiv:1805.12130.

[9] C.Alexandrou, S.Bacchio, M.Constantinou, J.Finkenrath, K.Hadjiyiannakou, K.Jansen, G.Koutsou, A.V.Aviles Casco, Phys. Rev. D 100, 014509 (2019).

[10] C. Alexandrou, K. Cichy, M. Constantinou, K. Jansen, A. Scapellato, F. Steffens, Phys. Rev. Lett. 121, 112001 (2018).

[11] X.Ji, Phys. Rev. Lett. 110, 262002 (2013).

[12] J. Fallica, Ph.D. thesis, Carnegie Mellon University (2017).

[13] M. Lüscher, Nucl. Phys. B354, 531 (1991).

[14] C. Morningstar, J. Bulava, B. Singha, R. Brett, J. Fallica, A. Hanlon, B. Hörz, Nucl. Phys. B924, 477 (2017).

[15] C.W. Andersen, J. Bulava, B. Hörz, C. Morningstar, Phys. Rev. D 97, 014506 (2018).

[16] S. Paul, G. Silvi, C. Alexandrou, G. Koutsou, S. Krieg, L. Leskovec, S. Meinel, J. Negele, M. Petschlies, A. Pochinsky, G. Rendon, S. Syritsyn, PoS(LATTICE2018)089.

[17] C. Andersen, B. Hörz, J. Bulava, C. Morningstar, in prep.

[18] B. Hörz, C. Andersen, J. Bulava, M. Hansen, D. Möhler, C. Morningstar, H. Wittig, in prep.

[19] C. Andersen, J. Bulava, B. Hörz, C. Morningstar, Nucl. Phys. B939, 145 (2019).

[20] T. Iritani, S. Aoki, T. Doi, T. Hatsuda, Y. Ikeda, T. Inoue, N. Ishii, H. Nemura, K. Sasaki, JHEP03, 007 (2019).

[21] A. Hanlon, A. Francis, J. Green, P. Junnarkar, H. Wittig, PoS LATTICE2018 (2018) 081. 